\section{Origins of life}

\section{A plausible route to the firstgenetic alphabet}

\section{Kristian Le Vay \& Hannes Mutschler}

\section{Understanding the prebiotic origins of the nucleic acids is a long-standing challenge. The latest experiments support the idea that the first nucleic acid encoded information using a mixed 'alphabet' of RNA and DNA subunits. See p.60}

The genetic polymers RNA and DNA are central to information storage in all biological systems, and as such form the core of most hypotheses about the origin of life. The most prominent of these theories is the 'RNA world' hypothesis, which posits that RNA was once both the central information-carrier and the catalyst for biochemical reactions on Earth before the emergence of life ${ }^{1}$. However, studies in the past few years (see ref. 2, for example) have suggested that the first genetic systems might have been based on nucleic-acid molecules that contain both RNA and DNA nucleotides, which then gradually self-separated into today's RNA and DNA. On page 60, Xu et al. ${ }^{3}$ offer fascinating experimental support for a mixed RNA-DNA world.

Primordial geochemical processes are thought to have led to the formation of the building blocks of nucleic acids - nucleotides and nucleosides (nucleotides that lack a phosphate group). Under suitable conditions, these building blocks polymerized and the resulting strands eventually replicated, without assistance from modern protein enzymes.

Workers from the same research group as Xu et al. had previously identified ${ }^{4}$ a network of reactions promoted by ultraviolet light that resulted in the synthesis of two of the standard nucleosides found in RNA: uridine $(\mathrm{U})$ and cytidine (C), which are collectively known as pyrimidines (Fig. 1). These reactions started from hydrogen cyanide $(\mathrm{HCN})$ and derivatives thereof, simple molecules thought to have been readily available on early Earth. Further studies and development of this reaction network raised the intriguing possibility that protein and lipid precursors could have arisen simultaneously alongside nucleosides ${ }^{5}-$ thereby providing three of the main types of molecule needed to make cells. However, a complementary route for the formation of the other two standard RNA nucleosides (adenosine and guanosine, known as the purines) using the same $\mathrm{HCN}$-based chemistry has remained elusive.

In the present work, Xu et al. revisited compounds produced as intermediates in the previously established reaction network $^{4}$ that synthesizes $U$ and $C$. They identified a pathway in which a key intermediate of pyrimidine-nucleoside synthesis, ribo-aminooxazoline (RAO; Fig. 1), can also be converted into two purine DNA nucleosides, deoxyadenosine $(\mathrm{dA})$ and deoxyinosine $(\mathrm{dI}$, which is not one of the standard nucleosides found in modern DNA). Crucially, these DNA nucleosides can form base pairs with $\mathrm{U}$ and C. The four nucleosides $-\mathrm{U}, \mathrm{C}, \mathrm{dA}$ and $\mathrm{dI}-$ therefore constitute a complete 'alphabet' that could have encoded genetic information in nucleic acids in a prebiotic RNA-DNA world.

Importantly, the synthesis of $\mathrm{dA}$ and $\mathrm{dI}$ can occur in parallel with that of $\mathrm{U}$ and $\mathrm{C}$, producing mixtures of the four products in yields and ratios suitable for the construction of a genetic system. This mutual compatibility of the two synthetic pathways increases the plausibility of the reaction network as a prebiotic system - if the two syntheses were incompatible, then geological scenarios would need to be contrived to explain how they could have been separated into different pools to enable the chemistry to occur, and then

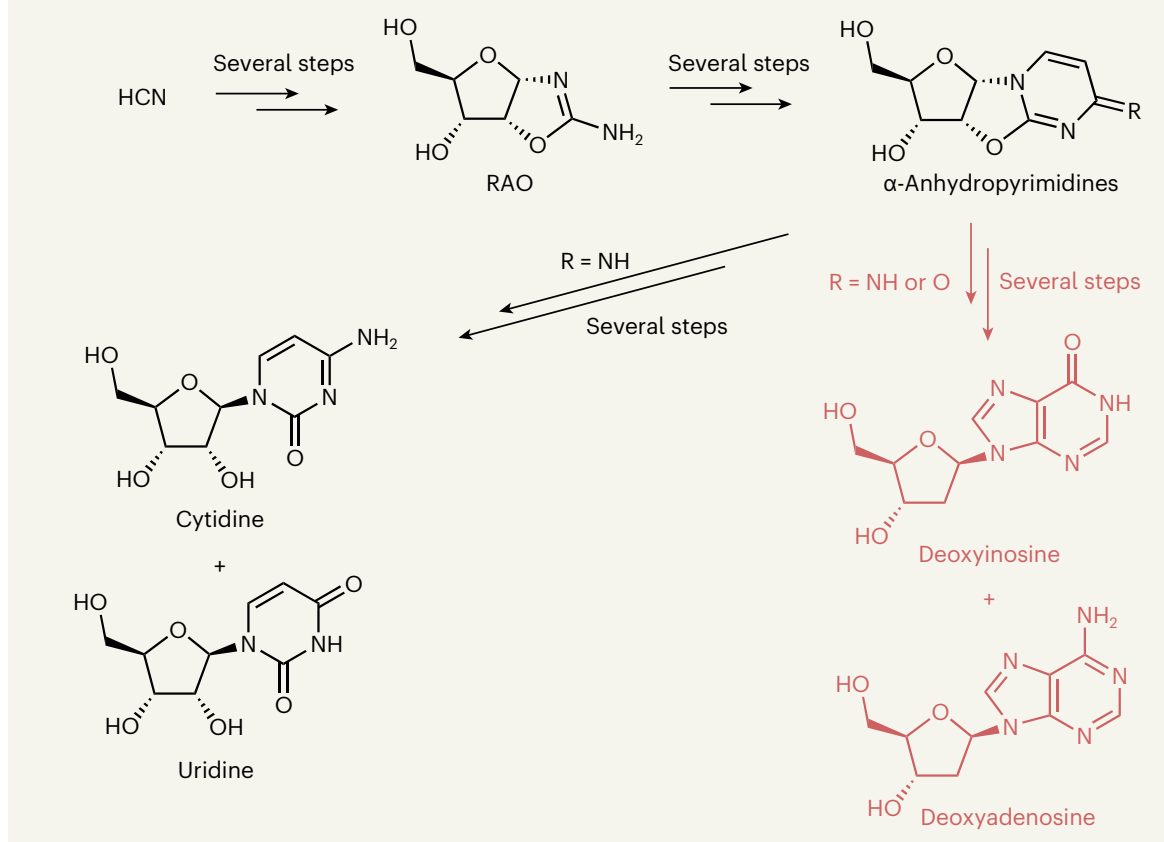

Figure 1 | A reaction network that produces both DNA and RNA subunits. It was known that a network of chemical reactions produces the RNA subunits cytidine (C) and uridine (U), under conditions that could have occurred on prebiotic Earth ${ }^{4}$. The network starts from hydrogen cyanide $(\mathrm{HCN})$ and proceeds through an intermediate called ribo-aminooxazoline (RAO). Xu et al. ${ }^{3}$ now report that compounds known as $\alpha$-anhydropyrimidines produced in the pathway to $C$ and $U$ can also be converted in parallel into the DNA subunits deoxyinosine $(\mathrm{dI})$ and deoxyadenosine $(\mathrm{dA})$. These subunits can form base pairs with $\mathrm{C}$ and $\mathrm{U}$. The four subunits $-\mathrm{C}, \mathrm{U}, \mathrm{dI}$ and $\mathrm{dA}$ - therefore constitute a complete genetic 'alphabet' that might have been used to encode biological information on early Earth. 
combined to enable the formation of hybrid RNA-DNA molecules. Notably, under certain reaction conditions, $U$ and $C$ can survive only in the presence of the thioanhydropurine compounds that act as direct precursors of dA and dI.

Many organic molecules can be produced as left- and right-handed versions, known as enantiomers, which are mirror images of each other. However, modern nucleotides and their building blocks all take the same enantiomeric form. One of the main difficulties in origins-of-life research is to explain how single enantiomers could have been generated from simple precursor molecules that have no handedness and which could have formed on prebiotic Earth. Xu and colleagues' purine synthesis is attractive in this respect, because it is highly selective for the enantiomers and other isomers of nucleosides observed in modern biology.

Alternative routes have been reported for the combined prebiotic synthesis of pyrimidine and purine nucleosides and nucleotides ${ }^{6,7}$. These routes require chemically and enantiomerically pure sugars to be used as starting materials, which poses the problem that other, often unknown, prebiotic processes would have been necessary to provide those starting materials ${ }^{8}$. By contrast, the enantioselectivity reported by Xu et al. derives from RAO, which can crystallize as a single enantiomer from reactions in which the starting materials are nearly racemic ${ }^{9}$ (that is, the starting materials consist of an almost equal mixture of enantiomers).

Nucleoside synthesis can also lead to products in which the nucleoside's base is attached to the sugar in the wrong orientation. In Xu and co-workers' synthetic pathway, a UV-induced chemical reduction occurs that leads to the strikingly selective destruction of these unwanted by-products, ultimately producing only the biologically relevant isomers of the purines. Given that early Earth was highly irradiated by UV, the remarkable selectivity of this reaction suggests a possible mechanism by which the total pool of potential nucleic-acid isomers was reduced to the subset of isomers observed today in nature.

Xu and colleagues' work supports a vision of early molecular evolution somewhat removed from the conventional 'pure' RNAworld hypothesis, and perhaps offers a more plausible route to the origin of life from mixed and complex chemical environments. Given the lack of 'chemical fossils', and the uncertainty over the exact conditions and chemistry that occurred on early Earth, it is impossible to say which chemical pathways actually took place. Instead, we must ensure that proposed systems conform as closely as possible to our understanding of what could realistically have happened on prebiotic Earth - not just the chemistry, but also the overall complexity of the reaction networks and their compatibility with other processes.

In the current work, the authors show that the four nucleosides can indeed be produced through processes that could reasonably be expected to have occurred on early Earth (such as hydrolysis, drying and UV irradiation), and provide plausible synthetic pathways that could supply the reactions with their required starting materials. However, as for all prebiotic syntheses, it remains hard to envisage the actual microenvironment that could have supported the many specific chemical transformations required to produce the building blocks of life in quantity.

Nevertheless, $\mathrm{Xu}$ and colleagues' work impressively demonstrates how a complete genetic alphabet might have arisen. Regardless of whether we think that life developed from RNA alone, or from more-complex mixtures of nucleic acids, systems-level thinking to find mutually compatible prebiotic chemical pathways will be crucial for developing truly plausible models of the first stages of life's emergence.

\section{Kristian Le Vay and Hannes Mutschler} are in the Biomimetic Systems research group, Max Planck Institute of Biochemistry, Martinsried 82152, Germany.

e-mail: mutschler@biochem.mpg.de

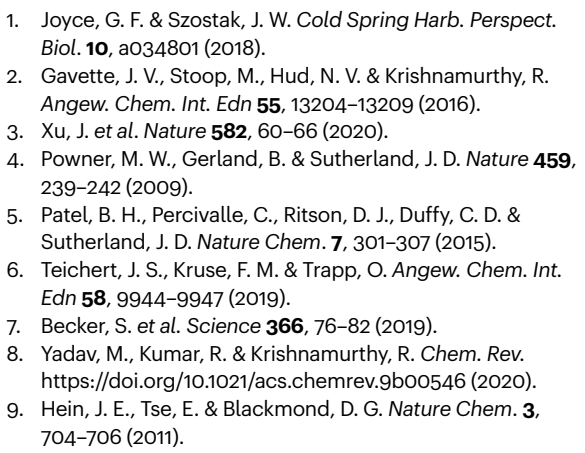

Neurodegeneration

\begin{abstract}
Ping Fang \& Elaine Y. Hsiao
disease. Analysis of a mouse model of the neurodegenerative condition amyotrophic lateral sclerosis offers insight into how gut bacteria might contribute to this illness. See p.89
\end{abstract}

Animals have co-evolved with diverse communities of microorganisms that are integral to the development and activity of their immune and nervous systems ${ }^{1}$. Alterations in the composition and function of the community of gut microorganisms (termed the microbiota) are increasingly being implicated in neurological disorders that involve neuroinflammation, including multiple sclerosis $^{2}$, autism spectrum disorder ${ }^{3}$ and Parkinson's disease ${ }^{4}$. Studies are also emerging that link the gut microbiota to amyotrophic lateral sclerosis (ALS), a neurodegenerative disorder characterized by the progressive loss of motor neurons crucial for movement, speech and cognition. This devastating disease is usually fatal within a few years of diagnosis. On page 89 , Burberry et al. ${ }^{5}$ fill some gaps in our knowledge of how gut microbes might contribute to ALS, from studies of the condition in a mouse model. Their findings might help to shed light on how a gene linked to ALS called C9orf72 affects this disease.

Initial studies ${ }^{6,7}$ have shown that the gut microbiota of people who have ALS differ from those of unaffected individuals. A study of a mouse model of the disease, based on an ALS-associated mutation in the Sod1 gene ${ }^{6}$, has provided strong evidence that alterations in the microbiota can exacerbate neurodegeneration and drive early mortality. That study also identified microbes and microbial molecules that promote improved motor function and longer lifespan in the mice. It showed that the particular positive or negative effects observed might depend on differences in the microbes encountered in the animals' housing facility (termed a vivarium). Mouse models of inflammatory diseases have also revealed that the animals' environment has such an effect ${ }^{8}$.

Burberry et al. used a mouse model of ALS (Fig. 1) in which the animals have a mutant version of the gene C9orf72, resulting in a deficiency in the encoded C9orf72 protein (these mice also model a neurodegenerative condition called frontotemporal dementia). The authors observed that if the animals were reared in the Harvard University animal facility, they had a shorter lifespan, 